\title{
Long-term effects of surgical angiogenic therapy with fibroblast growth factor 2 protein
}

Marc Ruel, MD

Roger J. Laham, $M D^{\mathrm{b}}$

J. Anthony Parker, MD, PhD ${ }^{\mathrm{C}}$

Mark J. Post, MD, PhD

J. Anthony Ware, $M D^{d}$

Michael Simons, $M D^{b}$

Frank W. Sellke, MD ${ }^{a}$

From the Departments of Surgery, ${ }^{\mathrm{a}}$ Medicine (Angiogenesis Research Center), ${ }^{\mathrm{b}}$ and Radiology, ${ }^{\mathrm{c}}$ Beth Israel Deaconess Medical Center and Harvard Medical School, Boston, Mass; and the Department of Medicine, ${ }^{\mathrm{d}}$ Albert Einstein College of Medicine, Bronx, NY.

Read at the Eighty-first Annual Meeting of The American Association for Thoracic Surgery, San Diego, Calif, May 6-9, 2001.

Received for publication May 15, 2001; revisions requested Aug 22, 2001; revisions received Oct 10, 2001; accepted for publication Nov 20, 2001.

This work was funded in part by National Institutes of Health grants HL-53793 and HL-56993 (Dr Simons), HL-46716 (Dr Sellke), HL-63609 (Dr Laham), and M01RR0132 (Dr Laham). Dr Ruel is supported by research fellowship awards from the Heart and Stroke Foundation of Canada in partnership with the Canadian Institutes of Health Research.

Address for reprints: Frank W. Sellke, MD, Division of Cardiothoracic Surgery, Beth Israel Deaconess Medical Center, 110 Francis St, Boston, MA 02215 (E-mail: fsellke@caregroup.harvard.edu).

J Thorac Cardiovasc Surg 2002;124:28-34

Copyright (C) 2002 by The American Association for Thoracic Surgery

0022-5223/2002 \$35.00+0 $\quad \mathbf{1 2 / 6 / 1 2 1 9 7 4}$

doi:10.1067/mtc.2002.121974
Objective: The long-term effects of surgical fibroblast growth factor 2 therapy are examined.

Methods: In a randomized, double-blind study, fibroblast growth factor 2 (10 $\mu \mathrm{g}$ or $100 \mu \mathrm{g}$ ) or placebo ( $\mathrm{n}=8 \mathrm{each})$ was delivered in the ungraftable myocardial territory of patients concomitantly undergoing coronary artery bypass grafting. Patients were followed up to $32.2 \pm 6.8$ months postoperatively with clinical assessment and nuclear perfusion imaging.

Results: Baseline patient characteristics were similar between the 3 groups. There were 2 late deaths, one of pancreatic cancer and one of undetermined cause (both in the 100- $\mu$ g fibroblast growth factor 2 group). Two patients (both in the control group) underwent a total of 6 repeat cardiac catheterizations for recurrent coronary events. Mean Canadian Cardiovascular Society angina class improved at late follow-up from baseline in all groups $(P \leq .02)$; however, patients treated with either dose of fibroblast growth factor 2 had significantly more freedom from angina recurrence than those treated with placebo $(P=.03)$. Late nuclear perfusion scans revealed a persistent reversible or a new, fixed perfusion defect in the ungraftable territory of 4 of 5 patients who received placebo versus only 1 of 9 patients treated with fibroblast growth factor $2(P=.02)$. The overall sum of left ventricular stress perfusion defect scores was also lower in fibroblast growth factor 2-treated patients than in control subjects $(1.3 \pm 1.4$ vs $3.9 \pm 2.1$, respectively; $P=.04)$. A trend toward a higher late left ventricular ejection fraction was noted in fibroblast growth factor 2-treated patients $(55.1 \% \pm 14.6 \%$ vs $44.3 \% \pm 6.5 \%$, fibroblast growth factor $2-$ treated patients versus control subjects; $P=.12$ ).

Conclusions: These data suggest that surgical angiogenic therapy with sustainedrelease fibroblast growth factor 2 may result in a prolonged myocardial revascularization effect that could translate into clinical benefit.

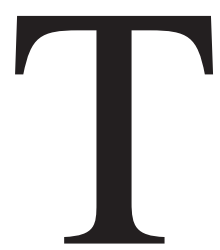

herapeutic angiogenesis is a promising modality for the treatment of patients with end-stage diffuse coronary artery disease for whom medical therapy has failed and who are not amenable to further direct coronary revascularization. Several delivery methods, including intravenous, intracoronary, or perivascular routes, have been used in animal and clinical studies to administer either protein- or gene-based formulations with variable success. ${ }^{1-5}$

Our group has previously reported the results of a phase I randomized controlled trial of local perivascular delivery of fibroblast growth factor 2 (FGF-2) in patients undergoing coronary artery bypass grafting $(\mathrm{CABG}) .^{3}$ In this study, patients in 
whom high-dose FGF-2 sustained-release capsules had been surgically implanted in an ungraftable territory at the time of $\mathrm{CABG}$ had complete relief from angina and showed significant improvements in stress nuclear perfusion defect size at 3 months' follow-up. These benefits were of greater magnitude than those observed in patients who received either placebo or low-dose FGF-2.

Although these early results appeared promising, there are to date little published long-term safety and efficacy data pertaining to any type of therapeutic angiogenesis modality. Theoretically, exogenous angiogenic agents could have the undesirable potential to stimulate intimal growth and destabilize atherosclerotic plaques, ${ }^{6,7}$ enable the development of unrecognized tumors, ${ }^{8}$ and promote other vascular pathologies, such as the formation of hemangiomas ${ }^{9}$ or the exacerbation of diabetic retinopathy. ${ }^{10}$ Although these potential complications have not been reported thus far in angiogenesis trials, their development would likely exhibit latency and escape clinical recognition until late follow-up is carried out.

Similarly, scant animal or clinical data exist that examine whether the effects of angiogenic therapies are long lasting. It is conceivable that the long-term efficacy of both proteinand gene-based delivery methods might be influenced, respectively, by the limited tissue half-life of protein formulations and by the high baseline levels of antibodies to adenoviral gene-delivery vectors found in the general population. ${ }^{11}$ Long-term data are also needed to better delineate the confounding role of a significant placebo effect observed in several clinical trials, as well as that of myocardial inflammation on the angiogenic response. ${ }^{4}$ To better ascertain some of these safety and efficacy issues, we conducted a long-term clinical and nuclear imaging follow-up study of patients previously enrolled in a phase I randomized controlled trial of perivascular FGF-2 delivered in an ungraftable myocardial territory during $\mathrm{CABG}$.

\section{Methods}

\section{Patient Selection}

The initial design of the study has been previously reported. ${ }^{3}$ The patient population consisted of patients undergoing CABG at Beth Israel Deaconess Medical Center (Boston, Mass) and Montefiore Medical Center (Bronx, NY). Inclusion criteria included the demonstration of an ischemic, viable myocardial territory supplied by a major coronary artery considered by both a cardiothoracic surgeon and an interventional cardiologist to be unamenable to bypass grafting or percutaneous intervention on the basis of its angiographic appearance. Ungraftability was also determined intraoperatively by a noninvestigator cardiac surgeon before confirmation of eligibility. Patients with clinically significant valvular disease, left ventricular ejection fraction of less than $20 \%$, serum creatinine level of greater than $2.5 \mathrm{mg} / \mathrm{dL}$, a history of malignancy in the previous 5 years, or a severe fixed nuclear perfusion defect in the ungraftable territory were excluded from the study.
The design and performance of the study were approved by the Food and Drug Administration under an investigational new drug protocol (BB-IND 5725), as well as by the Committee for Clinical Investigation at both institutions. The first patient was enrolled in September 1996 and the last patient in May 1998. Follow-up was completed in March 2001.

\section{Preparation and Delivery of FGF-2}

Sterile sustained-release heparin-alginate capsules containing either placebo or 10 or $100 \mu \mathrm{g}$ of human recombinant FGF-2 (Scios, Sunnyvale, Calif) were prepared as previously described. ${ }^{3,12,13}$ During aortic crossclamping, after completion of coronary bypass grafts to all revascularizable areas of the heart (including the routine construction of a left internal thoracic artery anastomosis to the left anterior descending artery and saphenous vein bypasses to other graftable territories) and failure to graft the target artery, intraoperative implantation of heparin-alginate capsules was performed. To this end, multiple linear incisions were made in the epicardial fat surrounding the target vessel, and 10 capsules containing either 1 of 2 doses of FGF-2 or placebo were inserted into the epicardial fat adjacent to the artery (2-3 capsules in each incision) and secured in place with a 6-0 polypropylene suture, as previously described. ${ }^{14}$ Patients were subsequently weaned from cardiopulmonary bypass, and routine closure was performed. The surgeon, other investigators, and patients were blinded to treatment-group assignments.

\section{Clinical Follow-Up}

Subsequent to in-hospital and early follow-up, the methods of which have been previously reported, ${ }^{3}$ patients were contacted yearly postoperatively to record the occurrence of major clinical events (death, myocardial infarction, recurrent angina, or repeat revascularization) and to determine their angina status according to the Canadian Cardiovascular Society (CCS) classification. In the third year after the operation, patients were brought back to their study center for a history, physical examination, and laboratory evaluation that included complete blood count, serum chemistries, urinalysis, and rest electrocardiography. Clinical follow-up was complete, with a mean duration (for patients who survived the perioperative period) of $32.2 \pm 6.8$ months (range, 23-44 months).

\section{Nuclear Perfusion Imaging}

In addition to clinical follow-up, patients underwent dual-isotope studies with thallium (rest) and dipyridamole sestamibi (pharmacologic stress) before CABG (first 10 patients) or postoperatively before discharge from the hospital (last 14 patients) and 3 months later. Patients were subsequently invited to return for a late nuclear assessment in their third or fourth postoperative year, with the exception of one patient in the control group in whom a midterm scan performed for symptoms of recurrent myocardial ischemia had revealed a new fixed defect in the ungraftable territory. All late nuclear assessments were done with a gated SPECT protocol and interpreted by a single nuclear medicine physician (J.A.P.) blinded to the territory of implantation and treatment-group assignments. Stress and rest perfusion scans for all 3 time points were assessed during one reading session, and myocardial images were subdivided into 5 myocardial regions (septal, anterior, lateral, inferior, and apical). Each territory was given a score of 0 (no defect), 1 
TABLE 1. Baseline patient characteristics

\begin{tabular}{|c|c|c|c|}
\hline & $\begin{array}{l}\text { Placebo } \\
(\mathrm{n}=8)\end{array}$ & $\begin{array}{c}\text { FGF-2, } 10 \mu \mathrm{g} \\
(\mathrm{n}=8)\end{array}$ & $\begin{array}{c}\text { FGF-2, } 100 \mu \mathrm{g} \\
(\mathrm{n}=8)\end{array}$ \\
\hline Age (y) & $47.4 \pm 11.4$ & $58.8 \pm 10.3$ & $60.2 \pm 10.1$ \\
\hline Male/female (n) & $7 / 1$ & $4 / 4$ & $8 / 0$ \\
\hline Hypertension (n) & 4 & 3 & 4 \\
\hline Tobacco use (n) & 3 & 4 & 5 \\
\hline Cholesterol >200 mg/dL (n) & 4 & 3 & 5 \\
\hline Diabetes (n) & 4 & 3 & 5 \\
\hline Prior CABG (n) & 0 & 2 & 1 \\
\hline Three-vessel disease (n) & 7 & 8 & 8 \\
\hline Pre-CABG LVEF (\%) & $53.6 \pm 14.7$ & $53.0 \pm 11.3$ & $44.4 \pm 15.1$ \\
\hline
\end{tabular}

$L V E F$, Left ventricular ejection fraction.

(mild), 2 (moderate), or 3 (severe defect). For a given territory, a score difference of 1 or more observed between separate studies was considered to be reflective of a change in myocardial perfusion, and a given defect was considered to exhibit a reversible component if a score difference of 1 or more points between stress and rest perfusion images was noted at a given study.

Left ventricular ejection fraction was calculated from gated wall-motion images obtained approximately 45 minutes after dipyridamole administration. Late nuclear studies were completed between 23 and 44 months (mean, $30.6 \pm 9.0$ months) after operation in 5 of 7 surviving patients in the control group, 5 of 8 surviving patients in the $10-\mu \mathrm{g}$ FGF-2 group, and 4 of 6 surviving patients in the $100-\mu \mathrm{g}$ FGF-2 group.

\section{Statistical Methods}

Continuous data are expressed as means \pm SD. Ordinal data, such as angina class, are expressed as median (2.5th percentile, 97.5th percentile). Statistical analyses were performed with nonparametric methods, with data pertaining to patients who survived the perioperative period. Angina class data were longitudinally compared within each group by using a Wilcoxon signed-rank test, as well as between groups by using a Kruskal-Wallis test. Angina class and left ventricular ejection fraction data were also compared between the placebo and combined FGF-2 treatment groups by using the Wilcoxon rank-sum test. Bonferroni corrections were applied on all tests involving repeated measures. Postoperative freedom from angina recurrence was assessed with the KaplanMeier method and compared between placebo and combined FGF2-treated patients with a log-rank test. Dichotomous variables were compared with a Fisher exact test. All reported probability values are 2 -tailed, and $P$ values of less than .05 were considered statistically significant.

\section{Results}

\section{Patient Characteristics}

Of 78 patients screened for enrollment into the study, a total of 24 patients who met eligibility criteria and accepted to take part in it were confirmed at the time of the operation to have one ungraftable coronary artery supply in ischemic and viable myocardial territory. These patients were randomized intraoperatively to receive 10 heparin-alginate capsules containing either $10 \mu \mathrm{g}$ of FGF-2 ( $\mathrm{n}=8), 100 \mu \mathrm{g}$ of FGF-2 $(\mathrm{n}=8)$, or placebo $(\mathrm{n}=8)$. Preoperative patient characteristics are summarized in Table 1 . With the exception of older age in the groups of patients who received $10 \mu \mathrm{g}$ and $100 \mu \mathrm{g}$ of FGF-2 and a higher proportion of women in the 10- $\mu$ g FGF-2 group, no significant difference was observed between the study groups in any of the preoperative clinical parameters.

\section{Short-Term Results}

Previously reported short-term results are summarized in Table 2.3 The implantation territory corresponded to the right coronary artery distribution in 15 patients, the left circumflex distribution in 7 patients, and the diagonal system in 2 patients. There was no significant difference in the number of grafts performed or total operative time between the 3 groups. One patient in the 100- $\mu$ g FGF-2 group who had a preoperative left ventricular ejection fraction of $20 \%$ could not be weaned from cardiopulmonary bypass and died intraoperatively. At autopsy, all grafts were patent.

Postoperatively, one patient in the control group died 24 hours after the operation after an autopsy-documented saphenous vein graft occlusion and a new large myocardial infarction in the subtended territory. Two other patients (both in the control group) required intra-aortic balloon pump support after the operation (in one patient the intraaortic balloon pump was inserted preoperatively). Two patients (one in the control group and one in the 10- $\mu$ g FGF-2 group), who subsequently declined to undergo late nuclear perfusion studies, had new Q waves in the ungraftable myocardial distribution, and one patient in the $10-\mu \mathrm{g}$ FGF-2 group had a Q-wave myocardial infarction in a bypassed territory. Placement of heparin-alginate FGF-2 capsules did not significantly affect acute hemodynamic parameters, serum chemistries, hematologic and coagulation profiles, liver function test results, or urinalysis. ${ }^{3}$

\section{Clinical Follow-Up}

Two late deaths occurred in the 22 patients who survived the perioperative period, both of whom were in the $100-\mu \mathrm{g}$ 
TABLE 2. Short-term results

\begin{tabular}{|c|c|c|c|}
\hline & Placebo (n = 8) & $\begin{array}{c}\text { FGF-2, } 10 \mu \mathrm{g} \\
(\mathrm{n}=8)\end{array}$ & $\begin{array}{c}\text { FGF-2, } 100 \mu \mathrm{g} \\
(\mathrm{n}=8)\end{array}$ \\
\hline \multicolumn{4}{|l|}{ Implanted coronary distribution (n) } \\
\hline RCA & 3 & 7 & 5 \\
\hline LCX & 3 & 1 & 3 \\
\hline Diagonal & 2 & 0 & 0 \\
\hline No. of grafts (n) & $3.1 \pm 1.3$ & $3.0 \pm 0.9$ & $3.1 \pm 0.8$ \\
\hline Duration of the operation (h) & $2.9 \pm 0.6$ & $3.0 \pm 0.9$ & $3.2 \pm 1.2$ \\
\hline Operative death (n) & 1 & 0 & 1 \\
\hline Perioperative myocardial infarction (n) & 1 & 2 & 0 \\
\hline
\end{tabular}

$R C A$, Right coronary artery; $L C X$, left circumflex artery.

TABLE 3. CCS angina class at baseline, 6 months, and late follow-up

\begin{tabular}{|c|c|c|c|c|}
\hline & Placebo & FGF-2, $10 \mu \mathrm{g}$ & FGF-2, $100 \mu \mathrm{g}$ & $P$ value* \\
\hline \multicolumn{5}{|l|}{ Median CCS angina class $†$} \\
\hline Baseline & $3(2,4)$ & $3(2,4)$ & $3(2,4)$ & .58 \\
\hline $6 \mathrm{mo}$ & $2(0,4)$ & $1(0,2)$ & $0(0,2)$ & .03 \\
\hline Late F/U & $1(0,2) \ddagger$ & $0(0,1)$ & $0(0,1)$ & .11 \\
\hline$P$ value§ (last F/U vs baseline) & .02 & .01 & .01 & \\
\hline
\end{tabular}

F/U, Follow-up.

${ }^{*} P$ value applies to control subjects versus combined FGF-2-treated patient groups at same time points (Wilcoxon rank-sum test). tData expressed as median (2.5th percentile, 97.5th percentile).

$\ddagger$ Two patients in the control group underwent repeated percutaneous coronary angioplasties for recurrent angina between the 6-month and 3-year follow-up assessments.

$\S P$ value applies to class improvements within each patient group between baseline and late follow-up (Wilcoxon signed-rank test).

FGF-2 group $(P=.09)$. The first patient, a 72-year-old man with a history of severe peripheral vascular disease and renal insufficiency, had complete resolution of his angina postoperatively but died suddenly 24 months after the operation. There was no consent to autopsy. The second patient, a 68-year-old man, died of metastatic pancreatic adenocarcinoma 37 months postoperatively. This patient had undergone implantation of $100-\mu \mathrm{g}$ FGF-2 capsules in the distribution of an ungraftable right coronary artery.

Two patients (both in the control group) experienced recurrent unstable angina, for which they underwent a total of 6 repeat coronary angioplasty procedures. Angioplasty and stenting were successful in achieving revascularization of myocardial distributions subtending a failed saphenous vein bypass graft but could not successfully revascularize the ungraftable territory in either patient. The 2 patients went on to have electrocardiographic evidence of myocardial infarction in the ungrafted territory and were free of angina at late follow-up.

For the 22 patients who survived the perioperative period, data pertaining to angina severity and postoperative freedom from recurrent angina are presented in Table 3 and Figure 1, respectively. Although all groups of patients experienced functional benefits at late follow-up when compared with baseline values $(P \leq .02$ for any of the 3 groups,
Table 3), patients treated with either dose of FGF-2 had significantly more freedom from recurrent angina postoperatively than those treated with placebo $(P=.03$, Figure 1$)$. There was no significant difference in CCS class at late follow-up or freedom from angina recurrence between patients who were treated with capsules containing $10 \mu \mathrm{g}$ versus $100 \mu \mathrm{g}$ of FGF-2.

\section{Nuclear Perfusion Imaging}

Results of late nuclear perfusion follow-up scans are presented in Table 4. In the control group all scanned patients but one had either a persistent reversible defect or a new fixed defect in the ungraftable territory, which was in contrast to only 1 of 9 patients who had been treated with either dose of FGF-2 (one patient in the $10-\mu \mathrm{g}$ and none in the $100-\mu \mathrm{g}$ group, $P=.02$ ). The sum of stress perfusion defect scores for all myocardial territories was also lower in FGF$2-$ treated patients than in control subjects $(P=.04)$. A trend toward a higher late left ventricular ejection fraction was noted in patients who were treated with FGF-2 versus that seen in control subjects by a magnitude of approximately $10 \%$ in ejection fraction units $(P=.12)$. No statistically significant difference was observed between the low-dose and high-dose FGF-2-treated groups in any of the above parameters. 


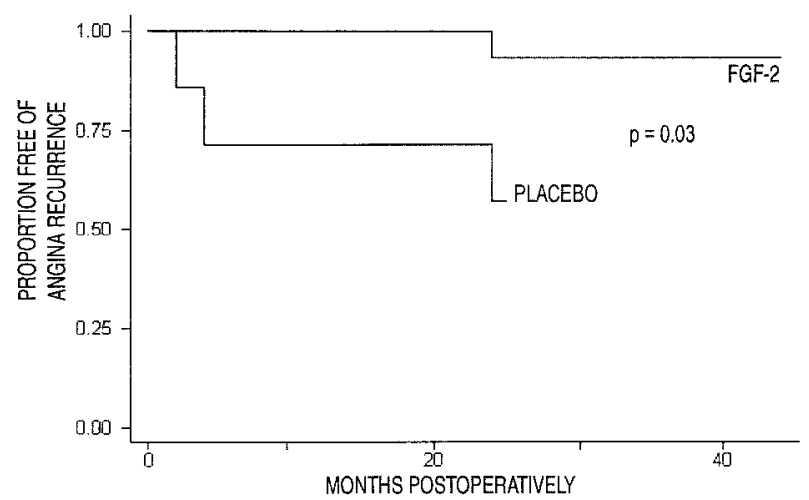

Figure 1. Freedom from recurrent angina by treatment group from the time of CABG. Patients treated with either dose of FGF-2 had significantly less angina recurrence postoperatively than those who received placebo $(P=.03$, log-rank test $)$.

\section{Discussion}

In this study patients in whom an ungraftable territory was treated concomitantly to CABG with perivascular administration of sustained-release FGF-2 capsules experienced significantly greater freedom from angina recurrence than control patients at a mean follow-up approaching 3 years. On late nuclear imaging studies, all but one patient in the control group had either persistence of a reversible perfusion defect or evidence of a new fixed defect in the ungraftable myocardial territory, whereas this was observed in only 1 of 9 patients treated with FGF-2. The remaining FGF-2treated patients had disappearance of their ungraftable territory reversible perfusion defect and stability or decrease in the size of their fixed defect (when one was present) compared with that seen at baseline. FGF-2-treated patients also showed better late global left ventricular perfusion scores during pharmacologic stress. Finally, a trend toward higher left ventricular ejection fraction was noted in FGF-2-treated versus control patients at late follow-up. These results suggest that perivascular angiogenic therapy combined with CABG might play a role in alleviating ischemia and preserving the function of ungraftable, ischemic, and viable myocardial territories in the late postoperative period.

The occurrence of 2 late deaths in the high-dose FGF2-treated group, albeit not statistically significant, nonetheless constitutes a source for concern regarding the long-term safety of angiogenic therapy. Because there was no consent for one patient to undergo autopsy and another died as a result of a metastatic pancreatic cancer that was diagnosed postoperatively, the possibility that perivascular FGF-2 therapy might have contributed to these deaths, despite a highly specific tissue distribution, ${ }^{15}$ cannot be excluded.

\section{Previous Related Work}

No published clinical trial of angiogenic therapy currently exists that sheds light onto key issues of dose response, duration of effect, or long-term safety with this approach. Despite a number of limitations, our study provides some preliminary information in this regard. In the previously reported short-term results of this patient cohort, a significant and dose-dependent effect of FGF-2 therapy was suggested by the finding of decreased perfusion defect size on stress nuclear imaging in patients who had received highdose perivascular FGF-2. ${ }^{3}$ The 3-year results reported here also suggest persistence of an overall FGF-2-related effect, but a dose relationship was not observed, possibly because of the study's sample size limitations.

Short- and long-term results of intramyocardial angiogenic therapy with FGF-1 have been previously reported by another group. ${ }^{16,17}$ In this study FGF-1 was injected directly into the myocardium along a diffusely diseased left anterior descending coronary artery to which the left internal thoracic artery was also grafted. Patients were subsequently followed up 3 years postoperatively with clinical examination, echocardiography, and selective imaging of the left internal thoracic artery with digital subtraction angiography. Although a local increase in collateral blush was observed along the left anterior descending coronary artery, the study did not report functional assessments of myocardial perfusion, data pertaining to changes in CCS angina class or freedom from angina, or the cause or timing of late deaths.

The FIRST trial recently evaluated the effects of intracoronary FGF-2 therapy in a large, double-blind phase II study involving 337 patients. ${ }^{18}$ Ninety-day follow-up revealed a significant improvement in angina frequency in patients treated with $3.0-\mu \mathrm{g} / \mathrm{kg}$ FGF-2 but no significant change in ejection fraction or in the size of ischemic territories on nuclear perfusion imaging. It is conceivable that the effects of FGF-2 in the FIRST trial, along with those of vascular endothelial growth factor in the preceding VIVA trial, ${ }^{19}$ might have been somewhat compromised by the choice of intracoronary and intravenous delivery routes, which are nonspecific in their tissue distribution. As shown in a previous study from our group, total cardiac-specific activity of vascular endothelial growth factor at 1 hour after injection was only $0.88 \%$ for intracoronary and $0.26 \%$ for intravenous administration and further decreased to $0.05 \%$ and $0.04 \%$, respectively, at 24 hours. ${ }^{20}$ Moreover, concern remains that the use of these delivery routes might actually worsen atherosclerosis because angiogenic growth factors stimulate endothelial cell proliferation and could potentially result in plaque expansion or instability when present in the diseased coronary circulation. ${ }^{6}$ On the other hand, surgical perivascular delivery might carry the additional, although theoretic, advantage of promoting the development of subepicardial collaterals between ischemic and normally perfused myocardium potentiated at surgical implantation by targeting the transition zone between collateral-dependent and normally perfused myocardial territories. 
TABLE 4. Third-year nuclear perfusion imaging

\begin{tabular}{|c|c|c|c|c|}
\hline & Placebo & FGF-2, $10 \mu \mathrm{g}$ & FGF-2, $100 \mu \mathrm{g}$ & $P$ value* \\
\hline Completed scans & $5 / 7$ & $5 / 8$ & $4 / 6$ & \\
\hline Improved perfusion (ungraftable territory) & $1 / 5$ & $5 / 5$ & $2 / 4$ & .09 \\
\hline Reversible defect or new fixed defect (ungraftable territory) & $4 / 5$ & $1 / 5$ & $0 / 4$ & .02 \\
\hline Sum of rest defect scores (all territories) & $2.3 \pm 2.1$ & $1.1 \pm 1.4$ & $0.88 \pm 0.85$ & .16 \\
\hline Sum of stress defect scores (all territories) & $3.9 \pm 2.1$ & $1.5 \pm 1.9$ & $1.0 \pm 0.82$ & .04 \\
\hline LVEF (\%) & $44.3 \pm 6.5$ & $53.8 \pm 18.0$ & $56.8 \pm 11.6$ & .12 \\
\hline
\end{tabular}

LVEF, Left ventricular ejection fraction.

${ }^{*} P$ values apply to control subjects versus combined FGF-2-treated patient groups.

\section{Limitations}

This study has several limitations. Although clinical follow-up is complete, some patients did not undergo late nuclear study imaging and consequently cannot be accounted for in some analyses. Conclusions must also be tempered with the possibility that confounding factors, such as intrinsic myocardial collateralization caused by chronic ischemia, might have influenced group comparability or results. The performance of 6 repeat revascularization procedures on 2 control group patients has resulted in their complete relief from angina before the conduct of late follow-up and might have biased against the demonstration of additional functional or nuclear imaging benefits in the FGF-2-treated versus placebo groups. Finally, the potential confounding effects of CABG on angina severity, perfusion of the ungraftable territory, and ejection fraction are also inherent to the study design. In this regard the possibility exists that a bypass graft might significantly increase perfusion to a distant myocardial territory through pre-existing or newly formed collaterals, regardless of the effects of angiogenic therapy. Baseline nuclear perfusion imaging studies were performed shortly after CABG in a majority of patients, as opposed to preoperatively, to partially limit this confounding factor.

Despite the aforementioned shortcomings, we hope that this study might bring preliminary insight into the long-term safety and efficacy of perivascular angiogenic therapy. As the field of therapeutic angiogenesis evolves, its results will necessitate elucidation and confirmation through long-term animal studies and phase III clinical trials.

We thank Constantin Yiannoutsos, $\mathrm{PhD}$, for his statistical review of the manuscript, and Deanna Niemann, RN, for her help with the conduct of follow-up evaluations.

\section{References}

1. Sellke FW, Simons M. Angiogenesis in cardiovascular disease: current status and therapeutic potential. Drugs. 1999;58:391-6.

2. Rosengart TK, Lee LY, Patel SR, et al. Angiogenesis gene therapy: phase I assessment of direct intramyocardial administration of an adenovirus vector expressing VEGF121 cDNA to individuals with clinically significant severe coronary artery disease. Circulation. 1999:100:468-74
3. Laham RJ, Sellke FW, Edelman ER, et al. Local perivascular delivery of basic fibroblast growth factor in patients undergoing coronary bypass surgery: results of a phase I randomized, double-blind, placebo-controlled trial. Circulation. 1999;100:1865-71.

4. Simons M, Bonow RO, Chronos NA, et al. Clinical trials in coronary angiogenesis: issues, problems, consensus: an expert panel summary. Circulation. 2000;102:E73-86.

5. Post MJ, Laham R, Sellke FW, Simons M. Therapeutic angiogenesis in cardiology using protein formulations. Cardiovasc Res. 2001;49: $522-31$.

6. O'Brien ER, Garvin MR, Dev R, et al. Angiogenesis in human coronary atherosclerotic plaques. Am J Pathol. 1994;145:883-94.

7. Libby P, Sukhova G, Lee RT, Galis ZS. Cytokines regulate vascular functions related to stability of the atherosclerotic plaque. J Cardiovasc Pharmacol. 1995;25:S9-12.

8. Folkman J. Angiogenesis in cancer, vascular, rheumatoid and other disease. Nat Med. 1995;1:27-31.

9. Brown LF, Tognazzi K, Dvorak HF, Harrist TJ. Strong expression of kinase insert domain-containing receptor, a vascular permeability factor/vascular endothelial growth factor receptor in AIDS-associated Kaposi's sarcoma and cutaneous angiosarcoma. Am J Pathol. 1996; 148:1065-74.

10. Adamis AP, Miller JW, Bernal MT, et al. Increased vascular endothelial growth factor levels in the vitreous of eyes with proliferative diabetic retinopathy. Am J Ophthalmol. 1994;118:445-50.

11. Roivainen M, Viik-Kajander M, Palosuo T, et al. Infections, inflammation, and the risk of coronary heart disease. Circulation. 2000;101: 252-7.

12. Edelman ER, Mathiowitz E, Langer R, Klagsbrun M. Controlled and modulated release of basic fibroblast growth factor. Biomaterials. 1991;12:619-26.

13. Harada K, Grossman W, Friedman M, et al. Basic fibroblast growth factor improves myocardial function in chronically ischemic porcine hearts. J Clin Invest. 1994;94:623-30.

14. Sellke FW, Laham RJ, Edelman ER, Pearlman JD, Simons M. Therapeutic angiogenesis with basic fibroblast growth factor: technique and early results. Ann Thorac Surg. 1998;65:1540-4.

15. Edelman ER, Nugent MA, Karnovsky MJ. Perivascular and intravenous administration of basic fibroblast growth factor: vascular and solid organ deposition. Proc Natl Acad Sci U S A. 1993;90:1513-7.

16. Schumacher B, Pecher P, von Specht BU, Stegmann T. Induction of neoangiogenesis in ischemic myocardium by human growth factors: first clinical results of a new treatment of coronary heart disease. Circulation. 1998;97:645-50.

17. Pecher P, Schumacher BA. Angiogenesis in ischemic human myocardium: clinical results after 3 years. Ann Thorac Surg. 2000;69:1414-9.

18. Simons M, Annex BH, Laham RJ, Kleiman N, Henry T, Dauerman H, et al. Pharmacological treatment of coronary artery disease with recombinant fibroblast growth factor-2: double-blind, randomized, controlled clinical trial. Circulation. 2002;105:788-93.

19. Henry TD, Abraham JA. Review of preclinical and clinical results with vascular endothelial growth factors for therapeutic angiogenesis. Curr Interv Cardiol Rep. 2000;2:228-41. 
20. Laham RJ, Rezaee M, Post M, et al. Intracoronary and intravenous administration of basic fibroblast growth factor: myocardial and tissue distribution. Drug Metab Dispos. 1999;27:821-6.

\section{Discussion}

Dr Todd K. Rosengart (Evanston, Ill). I would like to congratulate the authors on a very nice presentation. I would like to emphasize the point that the author has made, which I think is critical, that although this is an encouraging study, the limitations of a small sample size and the confounding effects of coronary bypass cannot be discounted, and in fact, I think it is important to take these factors into account in terms of using caution in judging these results.

I think that taken together, however, with the growing clinical body of information that now includes more than a half-dozen trials looking at angiogenic therapy, several trends appear to be increasingly more obvious. In our own study looking at adenoviral-mediated transfer of vascular endothelial growth factor, again using an intramyocardial approach, we have follow-up beyond 1 year. The trend again appears to be that intramyocardial delivery appears to be well tolerated. In the small number of patients that we have looked at thus far, there do not appear to be safety or toxicity issues, either with a protein-based or a gene-based strategy. And furthermore, in our follow-up in our series out to 1 year and again in your series out to 3 years, there appear to be encouraging initial results in terms of efficacy in parameters, such as angina class and treadmill.

Importantly, especially in your examination of basic FGF, there is a significant contrast with intramyocardial delivery versus intracoronary or intravenous delivery. As you pointed out, the VIVA trial or the basic FGF trial with intracoronary delivery has shown no evidence of benefit or very limited evidence and certainly a significant profile in regard to toxicity. Going forward, I think that the studies that look at intracoronary and intravenous delivery need to be carefully distinguished from studies such as yours that look at intramyocardial delivery. Therefore, again, there are encouraging initial trends in these early trials.

I have 2 questions specifically for the authors. One is that you have obviously gone to great lengths in using heparin-alginate beads, with the concept of sustained delivery being important, and I would ask you whether you have any evidence suggesting in fact that sustained release is necessary with basic FGF. And the second question is in regard to methodology. SPECT analysis clearly being an important part of your study, it is important to consider that there was just one observer, and do you have any evidence in terms of variability of results and not reproducibility?

Dr Sellke. Thank you, Dr Rosengart. The sustained delivery is probably not necessary. We found that in animal models a single delivery is probably adequate. However, for optimal vessel development, a sustained delivery is probably necessary. The SPECT imaging was blinded to a single observer, and, to our surprise, we did find positive results. However, there is quite a bit of variability in this analysis of flow with SPECT imaging. Therefore in general you either have to have a large number of patients in the sample or some luck, as I think we had, demonstrating a positive benefit.

Dr John R. Benfield (Los Angeles, Calif). I enjoyed your presentation greatly. We recently had the pleasure of Dr Judah Folkman at UCLA when he gave a beautiful summary of angiogenesis research and its potential clinical applications. I came away from that with the understanding that in angiogenesis inhibitor therapy for cancer, current evidence suggests that it is symbiosis or the synergy between chemotherapy and angiogenesis inhibitors. Today you nicely listed some of the confounding factors in your study, and these included CABG. My question is, what about the potentially confounding factor of nonoperative treatment of coronary atherosclerosis? How do you propose to control for that?

Dr Sellke. Well, with concomitant coronary bypass, you are increasing perfusion to border territories, and therefore I think it is important to assess perfusion defects and the size of perfusion defects after the revascularization. If you have a no-option trial, and I think that is what you are alluding to, it is important to have good control. That is, you need to have patients continue on medical therapy in the control group to have a good assessment of a real angiogenic effect. Those trials are going to be difficult to construct and to complete, but I think that they are necessary.

Your point about cancer therapy with antiangiogenic drugs is interesting. We actually might be causing a regression of collateral growth with these antiangiogenic factors and vice versa. If you give proangiogenic factors systemically to relieve cardiovascular symptoms, you might actually be increasing the propensity of tumors to grow.

Dr Reida M. El Oakley (Singapore). A recent report (Celletti FL, Waugh JM, Amabile PG, Brendolan A, Hilfiker PR, Dake MD. Vascular endothelial growth factor enhances atherosclerotic plaque progression. Nat Med. 2001;4:425-9) showed that the overexpression of vascular endothelial growth factor is associated with exacerbation of atherosclerosis and expansion of atheromatous plaques. First of all, would you care to comment on this? And second, are you aware of any data on the effect of basic FGF on atherosclerosis?

Dr Sellke. You are very right that angiogenic therapy, either vascular endothelial growth factor or other growth factors, might increase the vascularity of atherosclerotic plaques, and, at least in theory, lead to plaque softening and an increased incidence of plaque rupture. However, I do not think that has really been demonstrated, either in patients or in animal models. And I am not aware that basic FGF has been associated with that; however, that is a theoretic possibility. 